\title{
A role of three-dimensional (3D)-reconstruction in the classification of lung adenocarcinoma
}

\author{
Maristela L. Onozato*, Veronica E. Klepeis, Yukako Yagi and Mari Mino-Kenudson \\ Department of Pathology, Massachusetts General Hospital, Harvard Medical School, Boston, MA, USA
}

\begin{abstract}
Background: Three-dimensional (3D)-reconstruction from paraffin embedded sections has been considered laborious and time-consuming. However, the high-resolution images of large object areas and different fields of view obtained by 3D-reconstruction make one wonder whether it can add a new insight into lung adenocarcinoma, the most frequent histology type of lung cancer characterized by its morphological heterogeneity.

Objective: In this work, we tested whether an automated tissue sectioning machine and slide scanning system could generate precise 3D-reconstruction of microanatomy of the lung and help us better understand and define histologic subtypes of lung adenocarcinoma.

Methods: Four formalin-fixed human lung adenocarcinoma resections were studied. Paraffin embedded tissues were sectioned with Kurabo Automated tissue sectioning machine and serial sections were automatically stained and scanned with a whole slide imaging system. The resulting stacks of images were 3D reconstructed by Pannoramic Viewer software.

Results: Two of the four specimens contained islands of tumor cells detached in alveolar spaces that had not been described in any of the existing adenocarcinoma classifications. 3D-reconstruction revealed the details of spatial distribution and structural interaction of the tumor that could hardly be observed by 2D light microscopy studies. The islands of tumor cells extended into a deeper aspect of the tissue, and were interconnected with each other and with the main tumor with a solid pattern that was surrounded by the islands. The finding raises the question whether the islands of tumor cells should be classified into a solid pattern in the current classification.

Conclusion: The combination of new technologies enabled us to build an effective 3D-reconstruction of resected lung adenocarcinomas. 3D-reconstruction may help us refine the classification of lung adenocarcinoma by adding detailed spatial/structural information to 2D light microscopy evaluation.
\end{abstract}

Keywords: Lung, adenocarcinoma, solid, micropapillary, classification, histology, 3D, automation

\section{Introduction}

Three dimensional (3D)-reconstruction of histology sections has been seen as 'a laborious and timeconsuming' technique. The time consumed to achieve the results turned them into a non-attractive technique,

\footnotetext{
${ }^{*}$ Corresponding author: Maristela L. Onozato, MD, PhD, Department of Pathology, Massachusetts General Hospital, 101 Merrimac St, Suite 820, Boston, MA 02114, USA. Tel.: +1 617643 7904; Fax: +1617643 7900; E-mail: likaono@gmail.com.
}

although the information that it may give could be extremely useful in prognostication and therapeutics like in cancer. Digitization of image data has brought a great development in 3D field especially in radiology and also with confocal microscopy images. In other words, digital images with defined $\mathrm{X}$ and $\mathrm{Y}$-axis greatly benefited with digital 3D imaging technology but the same could not be applied to physical images such as light microscopy images. Those require a lot more of technological assistance. They do not 
have $\mathrm{X}$ and $\mathrm{Y}$ orientation therefore they need special algorithms for alignment orientation. Moreover, paraffin sections are subject to distortion, shrinkage, folding and color differences due to different staining intensities that may influence the quality and reliability of the reconstructed images $[1,2]$. However, the recent advances in technology, such as a robotic sectioning system and 3D-reconstruction software, may enable us to build an effective and user-friendly 3Dreconstruction system for histology sections.

Lung cancer is the leading cause of cancer death in the world and adenocarcinoma is the most common histologic subtype [3]. The treatment and prognosis of lung cancer have been linked to the classification and staging of these tumors; however, the classification of adenocarcinoma has been challenging due to the heterogeneity of its morphology with resultant multiple histologic patterns. Recently the multidisciplinary team of the International Association for the Study of Lung Cancer (IASLC), the American Thoracic Society (ATS), and the European Respiratory Society (ERS) have collaborated to develop a new and uniform classification of lung adenocarcinoma in order to improve the correlation between histology and the other features including clinical profile, staging, imaging characteristics, molecular alterations, and prognostic and predictive markers [3]. This new classification recognizes 5 common patterns (i.e. lepidic, acinar, papillary, micropapillary and solid patterns) and 4 variants in lung adenocarcinoma, and individual tumors are classified into a predominant pattern after measuring each pattern present in the tumor with $5 \%$ increments [3]. It has been shown that the classification, using a three-tiered system, correlates with patient prognosis in resected Stage I adenocarcinomas [4]. However, it is not infrequent that we encounter resected lung adencarcinomas exhibiting a morphology that is not easily classified according to the IASLC/ATS/ERS classification. For instance, we occasionally observe islands of tumor cells detached in alveolar spaces that are distinct from a micropapillary pattern $[5,6]$, and the clinical significance of these isolated tumor islands and their association with the other patterns are unknown.

The objectives of this study are twofold: (1) to build an easy and effective 3D-reconstruction method for physical images of resected lung adenocarcinoma combining new technologies, i.e. an automated sectioning system, whole slide imaging technology and 3D-reconstruction software; (2) to evaluate whether $3 \mathrm{D}$ imaging would deepen our understanding of the adenocarcinoma morphology and contribute to the improvement of the classification of lung adenocarcinoma.

\section{Materials and methods}

Four $10 \%$ formalin-fixed surgically resected stage I lung adenocarcinomas were included. These stage I patients had not received adjuvant chemotherapy, therefore this study allowed us to analyze the original histologic features without therapeutic effects. Tissues were embedded in high melting point-paraffin (hard paraffin, $58^{\circ} \mathrm{C}$ ) and automated sectioned. The study was approved by the Massachusetts General Hospital Institutional Review Board.

\subsection{Automated sectioning}

The three major potential sources of artifacts in 3D-reconstruction of histology sections are misalignment, shape distortion, and staining variation [2]. A robotic sectioning system was selected to test whether it could minimize these setbacks. Serial sections $(3 \mu \mathrm{m})$ were obtained with Kurabo AS-200S Automated Tissue Sectioning System (Kurabo Industries, Osaka Japan). Briefly, this robotic system consists of consecutive steps monitored by internal sensors. It automatically trims the tissue blocks, sections and mounts the tissue on the slide glass [7]. The advantages of this robotic sectioning system is that each section will have the same thickness, will be positioned with the same coordinates on the glass slide and the risk of section loss is lower than with manual section.

Two hundred slides from each block of approximately $1.0 \times 1.0 \times 1.0 \mathrm{~cm}$ were sectioned and 50 to 100 serial sections were randomly selected and hematoxylin-eosin (H\&E)-stained using a Leica MultistainerTM workstation (Leica Microsystems, Bannockburn, IL).

\subsection{Digital images and 3D-reconstruction}

Whole slide images (WSI) were obtained with Pannoramic Scan (3DHISTECH, Budapest, Hungary), using $20 \times$ objective magnification $(0.33 \mu \mathrm{m} /$ pixel $)$. Sets of 50 serial images without interval were selected and 3D images were constructed using Pannoramic Viewer 1.14.50 (3DHISTECH). 
Briefly, fine realignment of WSI was performed using Panoramic Viewer 1.14.50 using a Windows $\mathrm{XP}$ platform in a 34MB $2.46 \mathrm{~GB}$ of RAM hard disk computer. This software allows automatic stack montage with minimal intervention from the user except for image alignment and correction and color adjustments when necessary. The alignment could be either automatically or manually performed although manual alignment was preferred to get perfectly matched stacks. Images were stacked and a primary 3D platform was built, evaluated and selected areas were subtracted to build new platforms. Workflow is illustrated in Fig. 1. The whole image stacks were recorded and screen shots were taken and saved as JPEG files.

\section{Results}

Automated serial sectioned slides showed remarkable consistency of orientation and thickness facilitating the alignment process. In order to create 3D-reconstruction that was proportionally accurate, no slide was skipped and complete sets of serially sectioned slides were included. 'No-slide-skipping' is important because the software that reconstructs the 3D object considers the neighbors of the missing slide as serial slides, leading to distortion in the proportions of the 3D-reconstruction. Panoramic Viewer software showed a very user-friendly interface as it allowed to build and align stacks of images and to visualize the
A

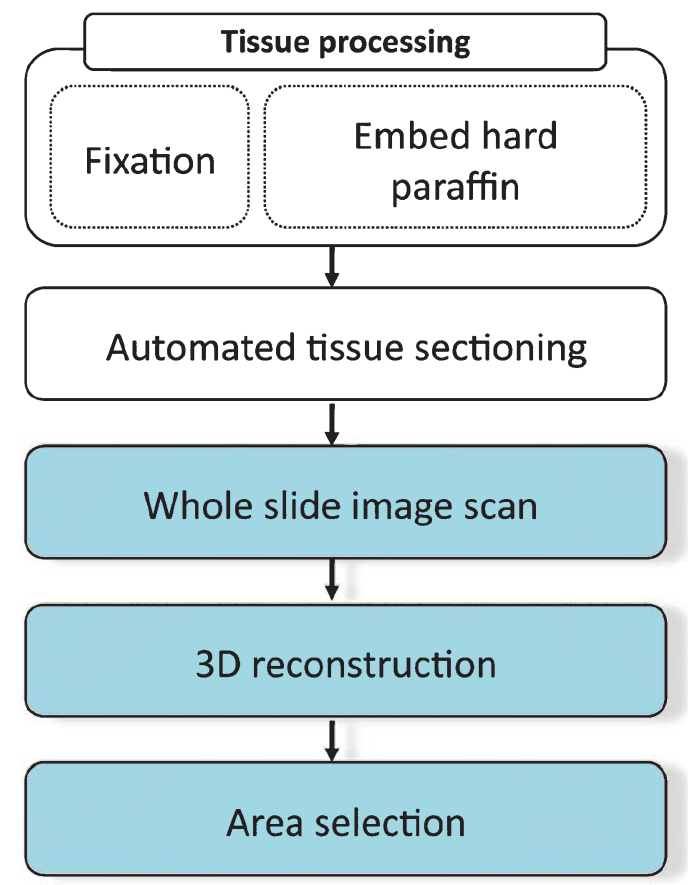

B

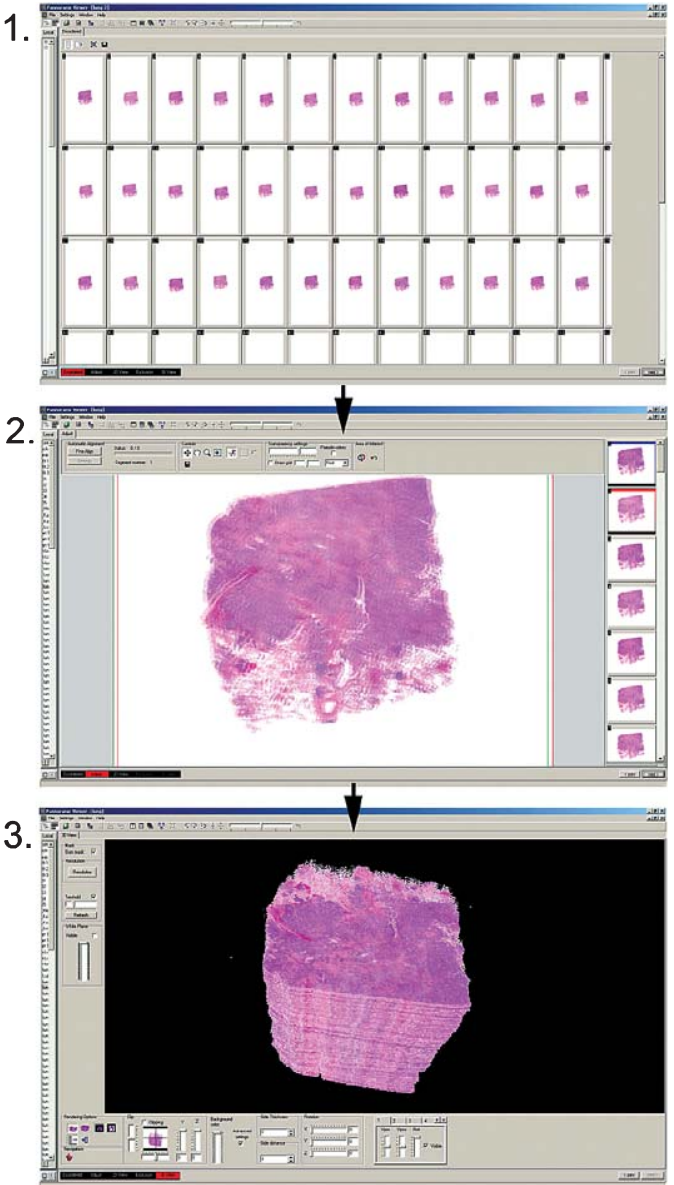

Fig. 1. Workflow for 3D-reconstruction. Tissue blocks are processed to be serial sectioned by the automated sectioning system. Once slides are sectioned and stained, they are scanned and whole slide images are obtained (A). With the 3D feature of the inbuilt software, stack of images are constructed (B-1) and aligned (B-2), imperfections (tissue debris) are selected and deleted from the stack and reconstruction is obtained (B-3). 


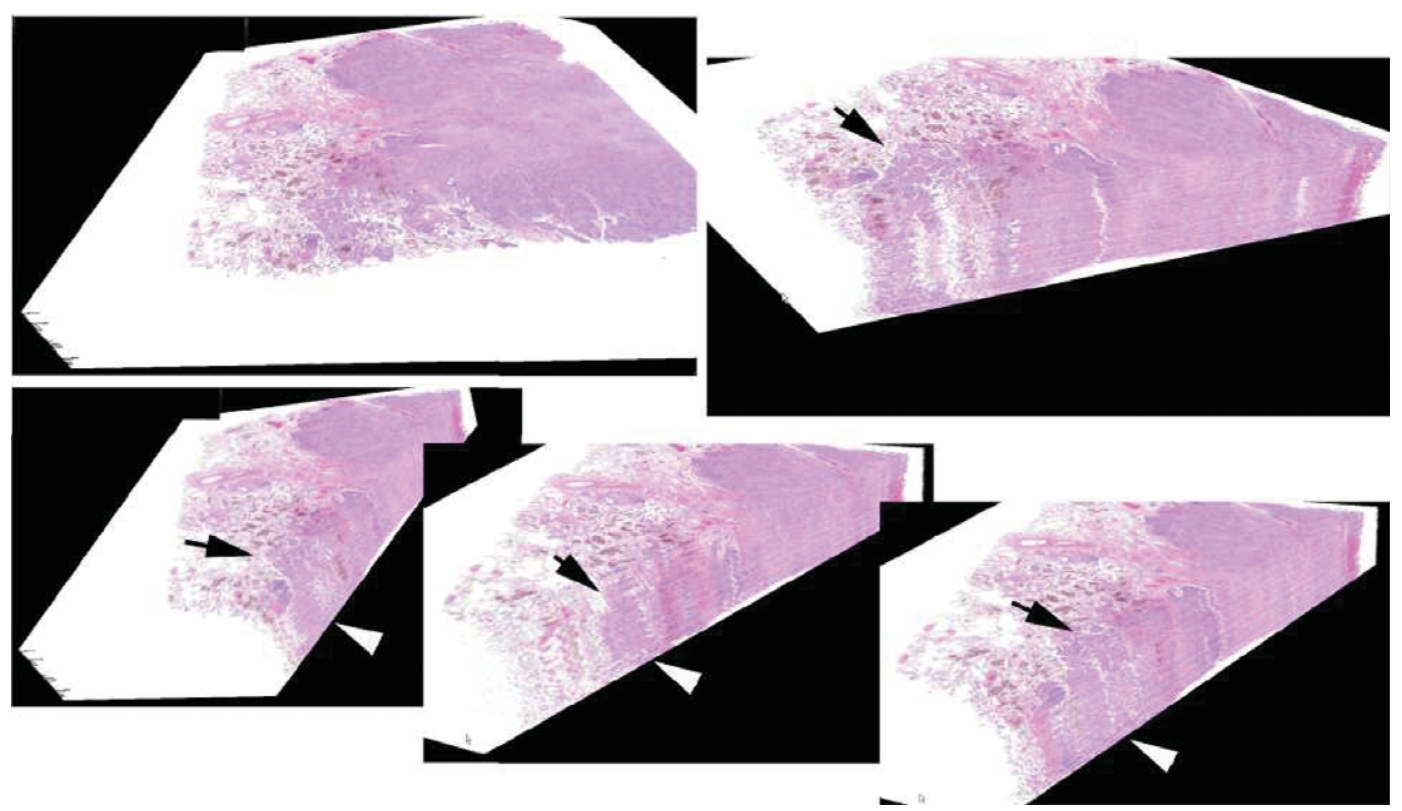

Fig. 2. 3D Images of Lung Adenocarcinoma. The panel shows images of 'island of tumor cells' in alveolar spaces. By 3D rendering these cells (arrow) extend into a deeper aspect of tissue, and are connected to other islands and/or the main tumor (arrow heads). These findings are difficult to identify with a $2 \mathrm{D}$ perspective.

entire data set in a single volume and to distinguish details in the microanatomy.

Of the four lung adenocarcinomas analyzed, two demonstrated detached, large nests of tumor cells within the alveolar spaces. These collections of cells were distinct from a micropapillary pattern that was defined as a pattern showing small clusters of tumor cells without a fibrovascular core [5, 6]. This 'islandlike' pattern inside the alveolar space can be observed in many cases of lung adenocarcinoma; however, its significance has remained unknown and it is not described in any of the existing histologic classifications. Interestingly, the 3D stacking showed connections between these tumor islands and those between the tumor islands and the main tumoral area with a solid pattern, although the islands of tumor cells appeared isolated within alveolar spaces on $2 \mathrm{D}$ view (Fig. 2). It is reasonable to say that non-slide-skipping methodology led to the identification of these connections.

\section{Discussion}

\subsection{D methods with automated systems}

The idea of 3D reconstruction of histology sections is not new but has not been considered a very attrac- tive tool for clinical purposes due to the effort and time involved in the reconstruction of physical images, though the information that it may provide could be significant.

Some basic factors are crucial for a reliable 3D visualization: good alignment, geometry congruence and staining consistency [1]. These factors also make the process very laborious. In this study we used a robotic sectioning machine followed by automated staining to minimize distortions inherent in formalinfixed paraffin-embedded tissue samples. The robotic sectioning machine was set to cut the sections with the same thickness and to place each section with the same coordinates on the slide glass therefore minimizing the effort of alignment and obtaining easily reproducible results with minimal or no loss of sections. However, even with the assistance of this robotic system, the image stack obtained from histology sections can never be completely isotropic and every 3D-reconstruction based on histology sections will contain some minor grade of artifacts and distortion due to the physical nature of each section.

We digitized the slides with a whole slide imaging scanner which provided us an overview of each slide so that we could build several image stacks from different areas covering a broad spectrum of histology in each 
specimen, and subsequently choose the ones that best showed features of interest. Major strengths of this type of 3D-reconstruction consist of high-quality 3D imaging and reliable tissue identification based on original 2D histology sections [1]. We used Pannoramic Viewer software that featured an easy-to-follow fine alignment tool and high-quality volume rendering $3 \mathrm{D}$ reconstruction. None of our reconstructions needed further image editing.

\subsection{D application in lung adenocarcinoma}

Recent trends have provided a growing interest in using these 3D images not only to visualize anatomy, but also to generate patient-specific anatomical models for treatment planning. These 3D models are useful for planning a surgical approach, for predicting the outcome of a planned surgery and for training and education [8].

We used our 3D model to have a better perspective on lung adenocarcinoma analyzing features that could not be clearly seen by $2 \mathrm{D}$ views of slide sections or could not be individualized by other imaging modalities (e.g. microCT and optical coherence tomography). We focused on the collections (islands) of tumor cells inside the alveolar space that have not been described in any of the preexisting lung adenocarcinoma classifications. Given the isolated nature of the tumor cells in alveolar spaces, some pathologists may classify them into a micropapillary pattern while some may not give diagnostic relevance to them. Interestingly, we found these islands of tumor cells in two of our specimens and a 3D perspective showed interconnections between the islands with larger ones presenting merging points with the main tumor mass with a solid pattern.

These interconnections raise the question as to whether the islands of tumor cells should be considered as the extension of solid components into alveolar spaces, which may dictate a more aggressive nature of the tumor, thus should be classified into a solid pattern, or should remain as unclassifiable components. In order to respond to the question, we are conducting another study to classify stage I lung adenocarcinomas in accordance with the ISALC/ATS/ERS International Multidisciplinary Classification with or without the island of tumor cells included in a solid pattern, and comparing the performance of the two classifications on predicting patient outcomes. It may also be impor- tant to evaluate whether the island of tumor cells itself has a prognostic value.

\section{Conclusion}

The combination of new technologies, i.e. a robotic sectioning system, whole slide imaging technology and 3D-reconstruction software enabled us to build an effective and user-friendly 3D-reconstruction system for histology sections. The 3D-reconstruction applied to lung adenocarcinoma resection specimens allowed us to evaluate architectural changes with the original color of staining and high-resolution not achievable by other modalities that are currently available in the medical field. 3D-reconstruction may be able to help us refine the classification of lung adenocarcinoma by adding detailed spatial/structural information to the assessment by light microscopy.

\section{Acknowledgments}

We thankfully acknowledge the technical assistance of Dr. Jie (Jenny) Zhao and Peggy Sherwood of the Wellman Center for Photomedicine, Histopathology Core Lab. (Boston, MA) and also for allowing us to use the facility. We thank the assistance of Gregory Mannheim. We gratefully acknowledge Kurabo Industries and 3D HISTECH for their technical support.

\section{References}

[1] S. Handschuh, T. Schwaha and B.D. Metscher, Showing their true colors: A practical approach to volume rendering from serial sections, BMC Dev Biol 10 (2010), 41.

[2] J. Streicher, W.J. Weninger and G.B. Muller, External marker-based automatic congruencing: A new method of 3D reconstruction from serial sections, Anat Rec 248 (1997), 583-602.

[3] W.D. Travis, E. Brambilla, M. Noguchi, A.G. Nicholson, K.R. Geisinger and Y. Yatabe, et al., International association for the study of lung cancer/american thoracic society/european respiratory society international multidisciplinary classification of lung adenocarcinoma, J Thorac Oncol 6 (2011), 244-285.

[4] A. Yoshizawa, N. Motoi, G.J. Riely, C.S. Sima, W.L. Gerald, M.G. Kris, B.J. Park, V.W. Rusch and W.D. Travis, Impact of proposed IASLC/ATS/ERS classification of lung adenocarcinoma: Prognostic subgroups and implications for further revision of staging based on analysis of 514 stage I cases, Modern Pathol 24 (2011), 653-664.

[5] H. Tsutsumida, M. Nomoto, M. Goto, S. Kitajima, I. Kubota, Y. Hirotsu, J. Wakimoto, M.A. Hollingsworth and S. Yonezawa, 
A micropapillary pattern is predictive of a poor prognosis in lung adenocarcinoma, and reduced surfactant apoprotein A expression in the micropapillary pattern is an excellent indicator of a poor prognosis, Mod Pathol 20 (2007), 638-647.

[6] N. Motoi, J. Szoke, G.J. Riely, V.E. Seshan, M.G. Kris, V.W. Rusch, W.L. Gerald and W.D. Travis, Lung adenocarcinoma: Modification of the 2004 WHO mixed subtype to include the major histologic subtype suggests correlations between papillary and micropapillary adenocarcinoma subtypes, EGFR mutations and gene expression analysis, Am J Surg Pathol 32 (2008), 810-827.
[7] M.L. Onozato, S. Hammnond, M. Merren and Y. Yagi, Evaluation of a completely automated tissue-sectioning machine for paraffin blocks, J Clin Pathol (September 2011). Online First, doi:10.1136.

[8] P. Kubik, G. Namyslowski, L. Turecka, C. Przeorek and N. Urbaniec, [Own experience in 3D reconstruction of the middle ear in spiral computer tomography], Otolaryngol Pol 54 (2000) 379-382. 


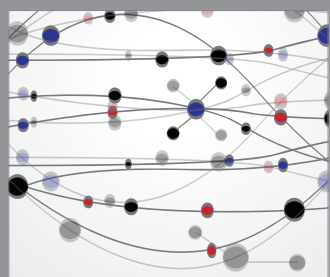

The Scientific World Journal
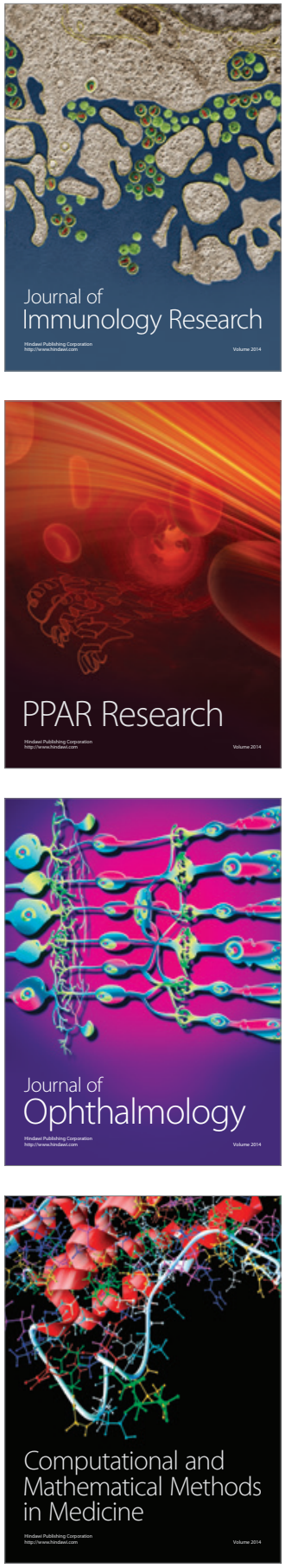

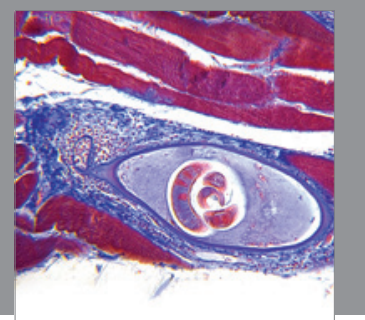

Gastroenterology

Research and Practice
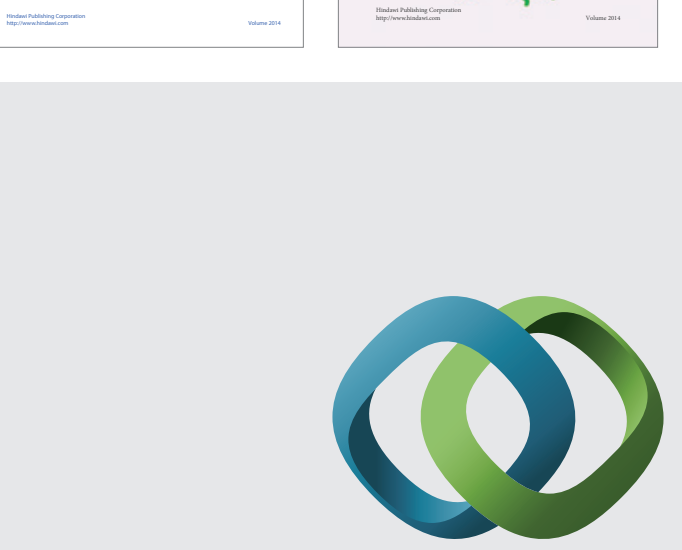

\section{Hindawi}

Submit your manuscripts at

http://www.hindawi.com
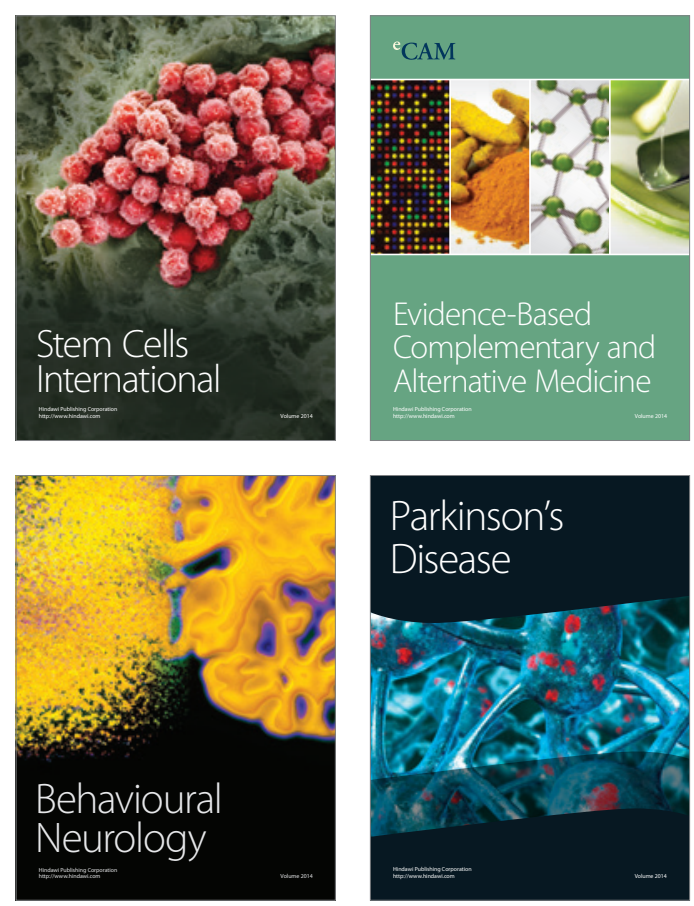

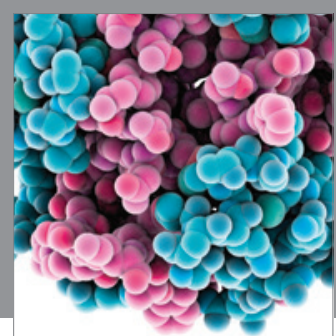

Journal of
Diabetes Research

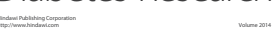

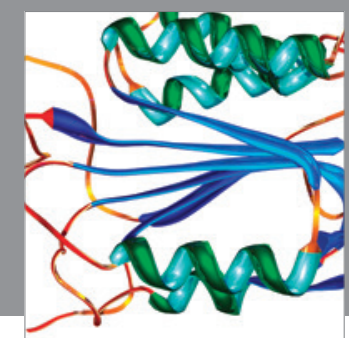

Disease Markers
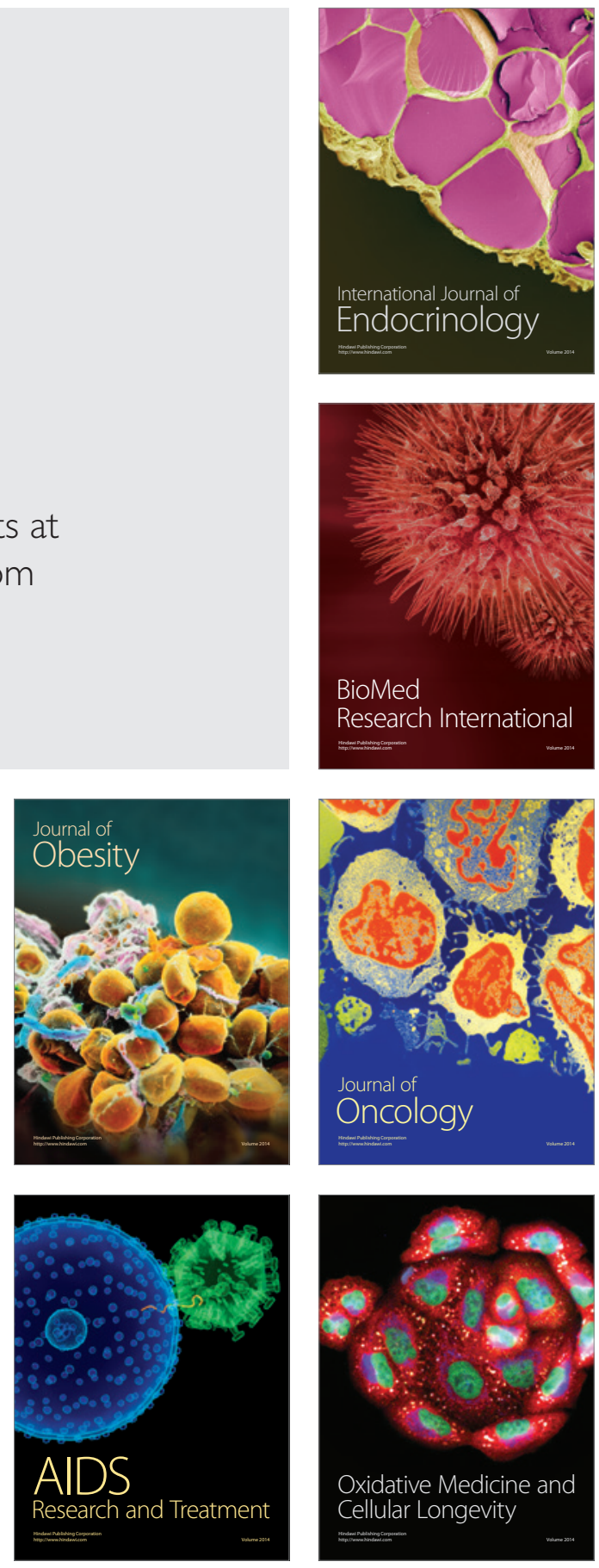